\title{
Inhaled corticosteroids and the risk of a first exacerbation in COPD patients
}

\author{
M.N. de Melo*, P. Ernst", S. Suissa\#
}

Inhaled corticosteroids and the risk of a first exacerbation in COPD patients. M.N. de Melo, P. Ernst, S. Suissa. (C) ERS Journals Ltd 2004.

ABSTRACT: The role of inhaled corticosteroids (ICS) in asthma is well established, but their benefit in the management of chronic obstructive pulmonary disease (COPD) is still controversial. The current study assessed whether ICS are effective in preventing a first exacerbation of COPD.

A cohort of newly treated COPD patients was formed from the Administrative Databases of Saskatchewan Health. The outcome was the occurrence of a first moderate or severe exacerbation from 1990-1999. Moderate exacerbations involved prescriptions for an antibiotic and an oral corticosteroid on the same day. Severe exacerbations were hospitalisations with a primary discharge diagnosis of COPD. A nested case-control design was used and matched on year of birth and cohort entry. Rate ratios (RR) were further adjusted for use of other medication and other confounders.

There were 995 exacerbations among 4,455 subjects. The rate of a first exacerbation was increased with any use of ICS in the year prior to the index date (RR: $1.27 ; 95 \%$ CI: 1.08-1.48) and with current use (RR: 1.51; 95\% CI: 1.22-1.87), and it increased with increasing daily doses of ICS.

Inhaled corticosteroids do not seem to be beneficial in reducing the risk of a first exacerbation of chronic obstructive pulmonary disease.

Eur Respir J 2004; 23: 692-697.
*Dept of Epidemiology and Biostatistics, McGill University, and ${ }^{\#}$ Division of Clinical Epidemiology, Pharmacoepidemiology Unit, Royal Victoria Hospital, McGill University Health Center, Montreal, Quebec, Canada.

Correspondence: S. Suissa, Division of Clinical Epidemiology, Royal Victoria Hospital, 687 Pine Avenue West, R.4.29, Montreal, Quebec, Canada, H3A $1 \mathrm{~A} 1$

Fax: 15148431493

E-mail: samy.suissa@clinepi.mcgill.ca

Keywords: Chronic obstructive pulmonary disease, cohort studies, drug therapy, inhaled corticosteroids

Received: May 52003

Accepted after revision: January 302004

This study was funded by the Canadian Institutes of Health Research (CIHR). The acquisition of the database was funded by Boehringer Ingelheim, GlaxoSmithKline and AstraZeneca. S. Suissa is the recipient of a Distinguished Investigator Award from CIHR. M. Melo is the recipient of a Scholarship from Associação Nacional das Farmácias, Portugal.

This study is based on de-identified data provided by the Saskatchewan Department of Health. The interpretation and conclusions contained herein do not necessarily represent those of the Government of Saskatchewan or the Saskatchewan Department of Health.
Inhaled corticosteroids (ICS) are currently the mainstay of adult asthma management and, although their benefit in chronic obstructive pulmonary disease (COPD) is still disputed [1, 2], they are also widely used in patients with COPD [3]. Certain similarities between asthma and COPD, e.g. the presence of both inflammatory and bronchospastic components, provide a rationale for the use of ICS in COPD patients. During the second part of the last decade several studies designed to investigate the efficacy of ICS in the treatment of COPD have been published, with most reporting negative results [4-14]. However, a few studies have suggested a beneficial effect of ICS in reducing the number and severity of exacerbations [4, 8, 12, 13]. More recently, a systematic review of randomised clinical trials of ICS in COPD showed a $30 \%$ reduction in the rate of exacerbations with the use of ICS [15]. However, none of the studies in this review had acute exacerbations as their primary end-point and the definition of an acute exacerbation varied or was not provided.

An acute exacerbation or "flare" of COPD is a frequent occurrence in COPD patients, although the definition of an exacerbation has varied between studies. It is characterised by an increase in symptoms often requiring a change in regular medication [16]. It is believed that bacterial infection is the underlying cause in as many as $70-75 \%$ of cases, with the remainder being attributed to viruses [17] and other causes [18]. Exacerbations have a considerable effect on morbidity and quality of life of COPD patients [19], and constitute an important cause of hospital admission and re-admission, with the consequent high burden on the healthcare system [19, 20]. In 1995, in the USA, total direct costs of inpatient and physician services for the treatment of acute exacerbations of COPD accounted for $\sim 1.6$ billion dollars [21] and in 1998, 662,000 hospitalisations ( $1.9 \%$ of all hospitalisations) were attributed to COPD [22].

In a cohort of COPD patients followed for $2.5 \mathrm{yrs}$ it was observed that 35 days after an exacerbation $\sim 25 \%$ of cases had not reached their baseline lung function and that by day 91 complete recovery of lung function had still not occurred in $7 \%$ of cases [12]. These results suggest a persistent deterioration in overall well-being with recurring episodes during the course of the disease. Hence, one important goal of treatment is to delay the time to a first exacerbation. Such a 
delay to first exacerbation has been shown with ICS in asthma [23]. This study assessed the effect of ICS in preventing a first exacerbation of COPD in a population-based cohort of COPD patients.

\section{Methods}

\section{Source of data and study population}

The primary source of data was the computerised Administrative Database of Saskatchewan Health. This database was developed to help coordinate the healthcare services provided to all residents of the province since 1975, with the exception of members of the Royal Canadian Mounted Police and Armed Forces. Two million people have been covered by this programme since it began. Dispensed prescriptions, use of healthcare services and vital status information are all recorded, and the information can be linked for each individual by means of a unique Health Service Number [24].

\section{COPD cohort}

The COPD cohort was initially formed from all individuals $\geqslant 55 \mathrm{yrs}$, who had received at least one bronchodilator medication (inhaled $\beta$-agonist, xanthine or ipratropium bromide) between January 1st, 1990 and December 31st, 1997. The current study restricted the cohort to individuals who had been dispensed more than three prescriptions on at least two different dates for these bronchodilators, in any annual period from January 1990 to December 1997. This makes it probable that treatment was more than occasional. Entry into the cohort occurred on the date of the third prescription. Subjects who had received $\beta_{2}$-agonists, ipratropium bromide, xanthines, cromolyn or nedocromil, nasal corticosteroids or ICS during the 5 yrs prior to cohort entry were excluded. This was done in an attempt to eliminate, as much as possible, patients with asthma, and to select subjects with first onset of regular drug therapy for COPD.

All patients in the cohort were followed from the date of cohort entry to the earliest of the following dates: December 31st, 1999, occurrence of a first COPD exacerbation, emigration from the province or death.

\section{Outcome}

The outcome of interest was the first exacerbation of COPD, moderate or severe, occurring after entry into the cohort. A moderate exacerbation was defined by prescriptions for a systemic antibiotic and an oral corticosteroid on the same day. A severe exacerbation was defined by hospitalisation with a primary discharge diagnosis of COPD (International Classification of Diseases, Ninth Revision (ICD-9) codes 490-492 and 496). The first of these exacerbations to occur was the outcome of interest and the date of its occurrence was considered the index date. As the present study was interested in a first COPD exacerbation, its cohort was restricted to individuals who had never had a recorded exacerbation, either moderate or severe, before entry into the cohort.

\section{Study design}

A nested case-control design was used with this study cohort. For each case all available controls were matched on the year of birth $( \pm 1 \mathrm{yr})$ and the year of entry into the cohort to control for secular trends in medical practice. In addition, controls had to be at risk of having the event at the time the case occurred, that is, they must have been alive and not had an exacerbation before this date. That date was also the index date for the matched controls.

\section{Exposure to inhaled corticosteroids}

The principal exposure of interest was current exposure to ICS, defined as dispensing of beclomethasone, flunisolide, fluticasone, triamcinolone or budesonide in the 2 months prior to the index date. Doses of ICS are expressed as beclomethasone equivalents, such that fluticasone $500 \mu \mathrm{g}$, budesonide $800 \mu \mathrm{g}$, flunisolide $2,000 \mu \mathrm{g}$ and triamcinolone $2,000 \mu \mathrm{g}$ were considered to be equivalent to $1,000 \mu \mathrm{g}$ of beclomethasone.

Given that there may be a prodromic phase of a COPD exacerbation, the current work identified dispensing of ICS occurring in the 15 days before index date. Current exposure was then analysed in two different ways: including the period of 15 days prior to the index date and excluding this period. The effect of having a prescription for ICS in the period 16-60 days before index and at least another one in the period of 61-365 days before index was also analysed. Finally, past use was considered whenever there was a prescription for an ICS dispensed in the 61-365 days before index, but not in the 60 days prior to the index date.

In order to ascertain the effect of different doses of ICS in preventing a first exacerbation of the disease, average daily doses of ICS over the previous year were calculated. Calculation of daily doses of ICS excluded hospitalisation days (for causes other than COPD), which occurred in the year prior to the index date. The Saskatchewan Prescription Database does not capture information on drugs used during hospitalisation. Therefore, the authors cannot exclude the possibility that some individuals might have been treated with inhaled, oral or intravenously administered corticosteroids while in the hospital.

\section{Statistical analysis}

All available controls were selected for each case and, therefore, descriptive statistics were weighted by the inverse of the number of controls in each matched set. This is equivalent to comparing cases and controls as if they were of equal number. Conditional logistic regression was used for matched case-control data to obtain crude and adjusted odds ratios as estimates of rate ratios (RR) of COPD exacerbations that were associated with the use of ICS. Adjustment was performed for severity of COPD, which is thought to be closely related to severe and/or more frequent exacerbations [25], as well as for concurrent diseases.

Severity of COPD was assessed by the following variables: number of prescriptions for oral corticosteroids; anti-allergic agents (cromolyn and nedocromil); inhaled, oral or nebulised $\beta_{2}$-agonists; xanthines; ipratropium bromide; and for systemic antibiotics. All medications were dispensed in the 12 months prior to the index date. Medications used during the 12-month period before the index date also served as proxy measures for comorbid disease. Comorbidities of interest were cardiac diseases, vascular diseases, diabetes, neurological disorders, psychiatric diseases and rheumatoid arthritis. Sex was addressed as a possible determinant and effect modifier.

Ninety-five per cent confidence intervals (CI) were computed for all RR. 


\section{Results}

The inception cohort of COPD patients was composed of 4,455 individuals, after 1,192 subjects were excluded because they had an exacerbation prior to cohort entry, that is, prior to the initiation of more than occasional bronchodilator use. All patients with a first COPD exacerbation after cohort entry were identified and matched with all the available controls. Between January 1st 1990, and December 31st 1999, there were 995 patients identified with a first exacerbation, of which 381 patients were identified with a moderate exacerbation and 614 with a severe exacerbation. The majority of the patients were males and mean age was 74 yrs.

Table 1 describes the distribution of the study subjects by selected characteristics, medications dispensed and possible confounding variables. The two groups were well matched, with the same mean age and similar follow-up time. Case

Table 1.-Characteristics of the study subjects

\begin{tabular}{|c|c|c|}
\hline & Cases & Controls $^{\#}$ \\
\hline Subjects $\mathrm{n}$ & 995 & 5416 \\
\hline Age mean \pm SD & $73.9 \pm 8.5$ & $73.9 \pm 8.2$ \\
\hline Male $\%$ & 58.6 & 51.0 \\
\hline $\begin{array}{l}\text { Mean days of follow-up } \\
\text { until index date }\end{array}$ & 897 & 921 \\
\hline \multicolumn{3}{|l|}{$\begin{array}{l}\text { Prescriptions in the previous } \\
12 \text { months of }\end{array}$} \\
\hline Oral corticosteroids & $26.0(0.85 \pm 2.14)$ & $8.1(0.23 \pm 1.16)$ \\
\hline Nasal corticosteroids & $5.4(0.16 \pm 0.90)$ & $5.8(0.16 \pm 0.90)$ \\
\hline Inhaled $\beta_{2}$-agonists & $74.6(4.98 \pm 5.55)$ & $52.7(2.53 \pm 3.77)$ \\
\hline Combined $\beta_{2}$ /ipratropium & $8.7(0.58 \pm 2.46)$ & $3.1(0.17 \pm 1.18)$ \\
\hline Oral $\beta_{2}$-agonists & $6.2(0.18 \pm 1.02)$ & $5.0(0.17 \pm 0.95)$ \\
\hline Inhaled ipratropium & $38.4(2.34 \pm 4.31)$ & $22.1(1.17 \pm 3.00)$ \\
\hline Anti-allergics & $2.1(0.09 \pm 0.81)$ & $1.3(0.05 \pm 0.64)$ \\
\hline Xanthines & $20.7(1.01 \pm 2.62)$ & $15.7(0.80 \pm 2.38)$ \\
\hline Antibiotics & $69.1(1.86 \pm 2.11)$ & $54.5(1.23 \pm 1.73)$ \\
\hline $\begin{array}{l}\text { Use of } \beta \text {-blockers and/or } \\
\text { benzodiazepines }\end{array}$ & 14.7 & 16.6 \\
\hline \multicolumn{3}{|l|}{$\begin{array}{l}\text { Comorbidity in year before } \\
\text { index date \% }\end{array}$} \\
\hline Cardiac disease & 39.8 & 34.9 \\
\hline Vascular disease & 52.7 & 52.7 \\
\hline Diabetes & 7.2 & 9.0 \\
\hline Psychiatric disease & 31.3 & 32.3 \\
\hline Neurological disorders & 7.1 & 7.1 \\
\hline Rheumatoid arthritis & 1.0 & 0.7 \\
\hline
\end{tabular}

Data are presented as percentages (mean $\pm \mathrm{SD})$ unless otherwise stated. \#: to account for case-control matching, all means and percentages for controls were weighted by the inverse of the number of controls in each matched case-control set; ${ }^{\uparrow}$ : any use during the month before index date. patients consisted of a higher proportion of males and were sicker than controls, as attested to by the higher mean number of prescriptions for respiratory medications and higher mean number of prescriptions for antibiotics dispensed during the 12-month period that preceded a first exacerbation (mean number of antibiotic prescriptions for cases 1.86 versus 1.23 for controls, $\mathrm{p}<0.05$ ). Current use of $\beta$-blockers and/or benzodiazepines was similar in cases and controls. In addition, case patients had increased cardiac disease $(p<0.025)$.

Table 2 summarises the results of the regression analyses for different time windows of ICS exposure. The adjusted RR for any use of ICS in the previous year, as compared with no use, was 1.27 (95\% CI: 1.08-1.48). Current exposure, excluding the 15-day period prior to the index date, as compared to no exposure, yielded an adjusted RR of 1.51 (95\% CI: $1.22-1.87)$. When the prodromic period was included, the adjusted RR did not change appreciably (adjusted RR=1.54, 95\% CI: 1.28-1.86). Moreover, past use of ICS and exposure in both the past and current periods were also associated with the risk of a first exacerbation (adjusted RR for past use 1.40 (95\% CI: 1.17-1.67), and adjusted RR for both current and past periods $1.52(95 \% \mathrm{CI}: 1.21-1.92))$. Furthermore, the risk of current exposure to ICS for a first exacerbation occurring in the first 2 yrs of follow-up was slightly greater than the risk of a first exacerbation occurring after 2 yrs from cohort entry (table 2).

Crude and adjusted RR for the effectiveness of various daily doses of ICS are displayed in table 3. The majority of cases and controls that took ICS used a daily dose of $\leqslant 500 \mu \mathrm{g}$ of beclomethasone or an equivalent dose of another

Table 2.-Matched rate ratios (RR) for chronic obstructive pulmonary disease exacerbations according to different patterns of inhaled corticosteroid (ICS) use

\begin{tabular}{|c|c|c|c|c|}
\hline Use in the past & Cases & Controls & Crude RR & $\begin{array}{l}\text { Adjusted RR } \# \\
\quad(95 \% \text { CI })\end{array}$ \\
\hline No use & 453 & 3232 & Reference & Reference \\
\hline Any use & 542 & 2184 & 1.77 & $1.27(1.08-1.48)$ \\
\hline Current $^{+}$ & 198 & 626 & 2.33 & $1.51(1.22-1.87)$ \\
\hline Past use $\mathrm{e}^{\S}$ & 359 & 1353 & 2.00 & $1.40(1.17-1.67)$ \\
\hline $\begin{array}{l}\text { Past and current } \\
\text { Current } \text { use }^{+}\end{array}$ & 171 & 524 & 2.44 & $1.52(1.21-1.92)$ \\
\hline$\leqslant 2 \mathrm{yrs}$ of $\mathrm{FU}$ & 528 & 2835 & 2.13 & $1.50(1.19-1.90)$ \\
\hline$>2 \mathrm{yrs}$ of $\mathrm{FU}$ & 467 & 2641 & 2.68 & $1.25(0.94-1.67)$ \\
\hline
\end{tabular}

Cases: $\mathrm{n}=995$; controls: $\mathrm{n}=5,416$; RR: rate ratio; FU: follow-up. ${ }^{\#}$ : adjusted for sex, number of prescriptions of inhaled $\beta_{2}$-agonists, combination of a $\beta_{2}$-agonist and ipratropium bromide, oral corticosteroids, antibiotics and ipratropium bromide, in the previous 12 months; " : any use in the previous 12 months; ${ }^{+}$: use of ICS 15-60 days before the index date; ${ }^{\S}$ : use of ICS 61365 days, but not in the 0-60 days before index date; ${ }^{f}$ : use of ICS in the period 16-365 days before index date.

Table 3. - Matched rate ratios (RR) for chronic obstructive pulmonary disease exacerbations by daily dose of inhaled corticosteroids

\begin{tabular}{|c|c|c|c|c|}
\hline Use in past year & Cases & Controls & Crude RR & Adjusted RR R $^{\#}(95 \% \mathrm{CI})$ \\
\hline None & 453 & 3232 & $\begin{array}{c}1.00 \\
\text { (reference) }\end{array}$ & $\begin{array}{c}1.00 \\
\text { (reference) }\end{array}$ \\
\hline$\leqslant 500 \mu \mathrm{g}$ & 350 & 1755 & 1.51 & $1.28(1.09-1.52)$ \\
\hline $501-1000 \mu \mathrm{g}$ & 118 & 328 & 2.70 & $1.56(1.19-2.04)$ \\
\hline $1001-1500 \mu \mathrm{g}$ & 56 & 76 & 5.48 & $2.32(1.54-3.48)$ \\
\hline$>1500 \mu \mathrm{g}$ & 18 & 25 & 6.36 & $2.94(1.46-5.89)$ \\
\hline Mean daily dose $\mu \mathrm{g}$ mean $\pm \mathrm{SD}$ & $258.0 \pm 440.7$ & $120.9 \pm 258.6$ & $3.42^{\circ}$ & $1.83(1.47-2.28)^{\bullet}$ \\
\hline
\end{tabular}

Cases: $\mathrm{n}=995$; controls: $\mathrm{n}=5,416 .{ }^{*}$ : adjusted for sex, number of prescriptions of inhaled $\beta$-agonists, combination of a $\beta_{2}$-agonist and ipratropium bromide, oral corticosteroids, antibiotics and ipratropium bromide, in the previous 12 months. ${ }^{\uparrow}$ : increase in risk is per $1,000 \mu \mathrm{g}$ increase in the daily dose of inhaled corticosteroids. 
ICS. The risk of a first exacerbation increased with increasing daily doses of ICS, varying from 1.28 for a daily dose of $\leqslant 500 \mu \mathrm{g}$ to 2.94 for a daily dose $>1,500 \mu \mathrm{g}$. This corresponds to an increase of $83 \%$ for every additional $1,000 \mu \mathrm{g}$ of beclomethasone or an equivalent dose of another ICS.

\section{Discussion}

The present study found that the use of ICS was not associated with a reduction in the risk of a first exacerbation of COPD. In fact, the risk appears to be slightly increased among users of ICS.

The current results are in accordance with the study of RENKEMA et al. [13], which showed that frequency and duration of exacerbations were not significantly different between the two active treatments (budesonide alone, budesonide and prednisolone) and placebo. PAgGiaro et al. [8] found similar results with fluticasone, administered for 6 months, with a similar number of exacerbations in the active and placebo groups, but less severe exacerbations in the fluticasone group. In contrast, results from the Lung Health Study and from the Inhaled Steroids in Obstructive Lung Disease (ISOLDE) trial suggested fewer exacerbations [4, 7] and fewer visits to general practitioners [4] among those receiving ICS. However, these were not the primary endpoints of these studies and the patients involved were younger.

The current authors results suggest no benefit of ICS, which is in opposition to the results of a recent systematic review that showed a $30 \%$ protective effect of ICS in preventing acute exacerbations of COPD [15]. However, this systematic review used information from studies where acute exacerbations were either not defined or where definitions varied substantially between studies. Furthermore, these studies combined were heterogeneous in other relevant characteristics, such as dose of ICS used and duration of follow-up. In particular, the authors did not study the first exacerbation, unlike the present work, to reduce the heterogeneity from varying disease severity and duration.

Confounding by indication is a major concern in observational studies of drug benefit, such as the current work, because the reason for prescribing a certain medication is often associated with the outcome of interest [26], introducing a bias. In theory, it would be possible to control for this, if one could measure with sufficient accuracy the effect of this confounder [26]. In studies using information from large drug databases, why a particular drug was prescribed at a particular time is not known, which makes it difficult if not impossible, to control completely for this bias. The current study used the frequency of prescriptions for respiratory disease dispensed during the year that preceded the exacerbation as a measure of severity. This assumes that sicker patients are more likely to receive more medications to treat their symptoms. Moreover, a matched design was used to control for effects of age and duration of disease, above and beyond the effect of severity of COPD. The present authors noticed that after controlling for sex and severity of the disease, the association of an increase in the risk of an acute exacerbation with the use of ICS was reduced significantly. However, residual confounding was probably present and remains the most likely explanation for the increased risk of a first exacerbation observed with increasing daily doses of ICS. With increasing severity, patients are more likely to be prescribed higher doses of ICS or are more likely to be prescribed these drugs more frequently.

Protopathic bias is also a concern in observational studies and is present when one attributes an outcome to an exposure that actually resulted from early signs and symptoms of the outcome under study [26]. The present study tried to avoid this bias by classifying as nonexposed the individuals who had been dispensed an ICS only during the prodromic phase, that is, during the 15-day period that preceded an exacerbation. However, in the present study, there was no difference in the risk of a first exacerbation in current users of ICS when the period of exposure was extended to allow exposure during the prodromic phase.

The authors chose to study a true first exacerbation because the period of time between exacerbations is believed to decrease with the progression of the disease, and full recovery after an acute exacerbation may not occur [12]. Therefore, if there was a benefit in using ICS it might be better perceived in the early stage of the disease. Any other exacerbation would be correlated with the preceding ones and the possible benefit of ICS might be difficult to assess. The current authors have previously reported a lack of benefit of ICS in preventing hospitalisations for an exacerbation of COPD among subjects who had been previously hospitalised for this same indication and had, therefore, progressed further in the natural course of COPD [27]. Moreover, by excluding subjects who had exacerbations before cohort entry, the current study can only generalise its results to patients who experience exacerbations after starting more than occasional treatment with bronchodilators.

The current study attempted to exclude patients who were likely to have asthma by excluding subjects who had received any respiratory drugs in the 5 yrs before cohort entry. This was done to isolate the effect of ICS in subjects with COPD since the authors have previously shown that ICS are effective at decreasing severe exacerbations of asthma [23]. It is unlikely that the current study was completely successful in excluding patients with asthma since this disease may have its onset after the age of $55 \mathrm{yrs}$. Inclusion of patients with asthma will have underestimated the increased risk of exacerbations of COPD that was observed with the use of ICS. Furthermore, the limitations due to assuming the current study patients had COPD based on the onset of more than occasional bronchodilator treatment after the age of $55 \mathrm{yrs}$ should be considered. This was made necessary by the lack of access to medical records with information on smoking and lung function, as is usually the case in database studies. While recurring therapy with bronchodilators may be prescribed for various reasons, it appears safe to assume that most of these subjects have a chronic respiratory disease, the most common at this age being COPD.

An individual was considered to be currently exposed if there was a dispensing of a prescription in the 60-day period prior to an exacerbation. Selection of a 60 -day period was based on two factors: 1) ICS are often prescribed in a high dose $\left(1,000 \mu \mathrm{g} \cdot \mathrm{day}^{-1}\right)$ for COPD patients; 2) the most commonly prescribed high-dose metered-dose-inhaler delivered 200 doses of $250 \mu \mathrm{g}$ each. Hence, it was estimated that a compliant patient would need to refill a prescription every 50 days. A period of 60 days was used to allow for some noncompliance and to allow for patients who were prescribed $<1,000 \mu \mathrm{g} \cdot \mathrm{day}^{-1}$.

This study used information from the Prescription Drug Database of the province of Saskatchewan, which is subject to several validation checks at the time a prescription is dispensed. In addition, submitting pharmacies routinely undergo audits [24]. However, in this database, information available represents only dispensed prescriptions and thus may not correspond entirely to medication used. This would result in a nondifferential misclassification of exposure with a consequent underestimation of the RR.

The results of this study depend in part on the definition of a moderate exacerbation. The authors defined a moderate exacerbation as a same day dispensing of an oral corticosteroid 
and antibiotic. This definition is consistent with that recently proposed during a workshop [16]. However, because there is still a dispute on whether the use of antibiotics in treating exacerbations of COPD is useful [28, 29], many physicians may not prescribe antibiotics if they are uncertain that the exacerbation has a bacterial aetiology. SEEMUNGAL et al. [12] followed a cohort of COPD patients for $2.5 \mathrm{yrs}$, and observed that treatment was started at a median of 3 days after onset of symptoms; prednisolone was administered in $27.3 \%$ of exacerbations; antibiotics in $85.6 \%$; and both an antibiotic and a oral corticosteroid were administered in only $25 \%$ of patients with an exacerbation [12]. Moreover, $12 \%$ of exacerbations were left untreated. Hence, it is likely that the cases in the current study represent an underestimate of all occurrences of exacerbations, in which case point estimates of RR may be too low. In addition, for some cases the outcomes may not be their first exacerbation, but only their first exacerbation that was treated with both an antibiotic and an oral corticosteroid on the same day. A strict definition was used in this study to avoid counting as exacerbations the dispensing of antibiotics for reasons other than COPD and, because of the difficulty, in some situations, of distinguishing between prescriptions of oral corticosteroid bursts for the treatment of exacerbations from dispensing of oral corticosteroids for daily regular therapy of COPD or other conditions. Moreover, this study did not try to assess mild exacerbations, which patients usually manage themselves without seeking the assistance of a physician, for instance, by increasing the frequency of administration of his bronchodilator [16]. This would have been difficult to capture in a database study. The accuracy of the information on severe exacerbations leading to hospitalisation is dependent on how well the codes of discharge diagnosis are entered. RAwSON et al. [30] have previously shown the diagnosis of COPD taken from records of hospital discharge in the Saskatchewan databases to be quite reliable.

The current results can only be generalised to patients $\geqslant 55 \mathrm{yrs}$, with the onset of more than occasional bronchodilator therapy for COPD who were not treated for COPD exacerbations as the initial presentation of their illness and who had not received respiratory medications for the previous 5 yrs. Therefore, patients with COPD who remain untreated or patients whose onset of regular therapy for COPD was before the age of 55 yrs were not included in the present study.

In conclusion, the current study suggests that inhaled corticosteroids are not efficacious in preventing a first exacerbation of chronic obstructive pulmonary disease. The small increase in risk is most likely due to residual confounding by indication, but it is unlikely that the remaining unmeasured confounding would mask a significant benefit of inhaled corticosteroids.

\section{References}

1. Calverley PM. Inhaled corticosteroids are beneficial in chronic obstructive pulmonary disease. Am J Respir Crit Care Med 2000; 161: 341-342.

2. Barnes PJ. Inhaled corticosteroids are not beneficial in chronic obstructive pulmonary disease. Am J Respir Crit Care Med 2000; 161: 342-344.
3. Jackevicius C, Chapman KR. Prevalence of inhaled corticosteroid use among patients with chronic obstructive pulmonary disease: a survey. Ann Pharmacother 1997; 31: $160-164$.

4. The Lung Health Study Research Group. Effect of inhaled triamcinolone on the decline in pulmonary function in chronic obstructive pulmonary disease. $N$ Engl $J$ Med 2000; 343: 1902-1909.

5. Pauwels R, Löfdahl C, Laitinen LA, et al. Long term treatment with inhaled budesonide in persons with mild chronic pulmonary disease who continue smoking. $N$ Engl J Med 1999; 340: 1948-1953.

6. Vestbo J, Sorensen T, Lange P, Brix A, Torre P, Viskum K. Long-term effect of inhaled budesonide in mild and moderate chronic obstructive pulmonary disease: a randomised controlled trial. Lancet 1999; 353: 1819-1823.

7. Burge PS, Calverley PM, Jones PW, Spencer S, Anderson JA, Maslen TK. Randomised, double blind, placebo controlled study of fluticasone propionate in patients with moderate to severe chronic obstructive pulmonary disease: the ISOLDE trial. BMJ 2000; 320: 1297-1303.

8. Paggiaro PL, Dahle R, Bakran I, Frith L, Hollingworth K, Efthimiou J. Multicentre randomised placebo-controlled trial of inhaled fluticasone propionate in patients with chronic obstructive pulmonary disease. International COPD Study Group. Lancet 1998; 351: 773-780, (published erratum Lancet 1998; 351: 1968).

9. Nishimura K, Koyama H, Ikeda A, et al. The effect of high-dose inhaled beclomethasone dipropionate in patients with stable COPD. Chest 1999; 115: 31-37.

10. Culpitt SV, Maziak W, Loukidis S, Nightingale JA, Matthews JL, Barnes PJ. Effect of high dose inhaled steroid on cells, cytokines, and proteases in induced sputum in chronic obstructive pulmonary disease. Am J Respir Crit Care Med 1999; 160: 1635-1639.

11. Weiner $\mathrm{P}$, Weiner $\mathrm{M}$, Azgad $\mathrm{Y}$. Inhaled budesonide therapy for patients with stable COPD. Chest 1995; 108: $1568-1571$.

12. Seemungal TAR, Donaldson GC, Bhowmik A, Jeffries DJ, Wedzicha JA. Time course and recovery of exacerbations in patients with chronic obstructive pulmonary disease. Am J Respir Crit Care Med 2000; 161: 1608-1613.

13. Renkema TE, Schouten JP, Koeter GH, Postma DS. Effects of long-term treatment with corticosteroids in COPD. Chest 1996; 109: 1156-1162.

14. Keatings VM, Jatakanon A, Worsdell YM, Barnes PJ. Effects of inhaled and oral glucocorticoids on inflammatory indices in asthma and COPD. Am J Respir Crit Care Med 1997; 155: 542-548.

15. Alsaeedi A, Sin DD, McAlister FA. The effects of inhaled corticosteroids in chronic obstructive pulmonary disease: a systematic review of randomized placebo-controlled trials. Am J Med 2002; 113: 59-65.

16. Rodriguez-Roisin R. Toward a consensus definition for COPD exacerbations. Chest 2000; 117: 398S-401S.

17. Sethi S. Infectious etiology of acute exacerbations of chronic bronchitis. Chest 2000; 117: 380S-385S.

18. Voelkel N, Tuder R. COPD: Exacerbations. Chest 2000; 117: 376S-379S.

19. Doll H, Grey-Amante P, Duprat-Lomon I, et al. Quality of life in acute exacerbation of chronic bronchitis: results from a German population study. Respir Med 2002; 96: 39-51.

20. Seemungal TAR, Donaldson GC, Paul EA, Bestall JC, Jeffries DJ, Wedzicha JA. Effect of exacerbation on quality of life in patients with chronic obstructive pulmonary disease. Am J Respir Crit Care Med 1998; 157: 1418-1422.

21. Niederman MS, McCombs JS, Unger AN, Kumar A, Popovian R. Treatment cost of acute exacerbations of chronic bronchitis. Clin Ther 1999; 21: 576-591.

22. Mannino DM. COPD: Epidemiology, prevalence, morbidity and mortality, and disease heterogeneity. Chest 2002; 121 : 121S-126S. 
23. Blais L, Suissa S, Boivin J, Ernst P. First treatment with inhaled corticosteroids and the prevention of admissions to hospital for asthma. Thorax 1998; 53: 1025-1029.

24. Downey W, Beck P, McNutt M, Stang M, Osei W, Nichol J. Health databases in Saskatchewan. In: Strom B, ed. Pharmacoepidemiology. Chichester, John Wiley \& Sons, Ltd, 2000; pp. 325-345.

25. Sohy C, Pilette C, Niederman MS, Sibille Y. Acute exacerbation of chronic obstructive pulmonary disease and antibiotics: what studies are still needed? Eur Respir J 2002; 19: 966-975.

26. Collet J, Boivin JF. Bias and confounding in pharmacoepidemiology. In: Strom B, ed. Pharmacoepidemiology. Chichester, John Wiley \& Sons, Ltd, 2000; pp. 765-784.
27. Bourbeau J, Ernst P, Cockcroft D, Suissa S. Inhaled corticosteroids and hospitalisation due to exacerbation of COPD. Eur Respir J 2003; 22: 286-189.

28. Anthonisen NR, Manfreda J, Warren CPW, Hershfield ES, Harding GKM, Nelson NA. Antibiotic therapy in exacerbations of chronic obstructive pulmonary disease. Ann Intern Med 1987; 106: 196-204.

29. Saint S, Bent S, Vittinghoff E, Grady D. Antibiotics in chronic obstructive pulmonary disease exacerbations: a meta-analysis. JAMA 1995; 273: 957-960.

30. Rawson NS, Malcolm E. Validity of the recording of ischaemic heart disease and chronic obstructive pulmonary disease in the Saskatchewan health care datafiles. Stats Med 1995; 14: 2627-2643. 